\title{
Direct measurement of circulation using ultrasound
}

\author{
H. Johari, W. W. Durgin
}

\begin{abstract}
Ultrasound time-of-flight methods employing counter-propagating ultrasonic pulses are utilized for the direct measurement of circulation in vortical flows. Two schemes are described here which involve either a single straight path or a closed path. Both techniques are shown to result in time differences, between the counter-propagating pulses around the path, linearly proportional to the circulation enclosed by the ultrasound path. The ultrasound methods of circulation measurement do not require calibration constants and can be non-invasive. The reliability of the closed path ultrasound method was assessed by comparing the measured circulation values with those calculated from the lift measurements of a NACA 0012 airfoil. Two examples are also presented where the closed path ultrasound method has been applied to the flow over a delta wing and a free-surface vortex in a cylindrical tank.
\end{abstract}

\section{1}

\section{Introduction}

Vortex-dominated flows can be characterized by the location and strength of the vortices contained in the flow field.

A measure of the aggregate vortex strength is the circulation around a closed path encompassing the vortex (vortices). Although the distribution of vorticity within the vortex is an important factor from the vortex dynamics perspective, knowledge of the integrated vortex circulation can be quite useful in a number of settings. For example, if the circulation distribution of swept wings can be obtained readily, it will reveal the loading distribution which, in turn, will set the structural design of such wings.

Computation of circulation has traditionally been performed by taking the line integral of the velocity around closed paths. Classical anemometry methods, i.e. hot-wire/film and LDV,

Received: 8 October 1997/Accepted: 23 April 1998

H. Johari, W. W. Durgin

Mechanical Engineering Department

Worcester Polytechnic Institute

Worcester, MA 01609, USA

Correspondence to: $\mathrm{H}$. Johari

The measurements presented here are a result of the work performed by several of our graduate students, K. J. Desabrais, J. D. Moreira, R. H. Smith, and F. J. Weber, over the past several years. Their contributions to the development of the ultrasonic hardware and the resulting data are hereby acknowledged. have been utilized for obtaining the velocity vectors in a flow field. Mapping of the entire flow field in this way is a time-consuming task due to the large number of data points needed. Furthermore, free vortices, which are subject to spatial movement, would not reveal their true strength since flow mapping by traditional anemometry methods relies on flow stationarity to justify the time-averaging of the data. Recent developments of the Particle Image Velocimetry (PIV) techniques have made whole field measurements a reality (Adrian 1986, 1991; Willert and Gharib 1991). The PIV methods have advanced to the point where an array of velocity vectors in an instant of time within a two-dimensional slice of the flow can be calculated. Although the sampling rate of PIV systems is typically limited to about $10 \mathrm{~Hz}$, instantaneous velocity field data can provide the spatial structure of the flow as well as the integral measures such as circulation or volume flow rates. However, the application of PIV methods to aircraft wings or other large scale industrial devices may not be a straight forward matter because large areas have to be illuminated by a pulsed laser light sheet and high resolution cameras are needed. Moreover, opaque flow fields preclude the use of optically-based diagnostic tools.

The possibility of measuring circulation directly and noninvasively using ultrasound was raised by Schmidt $(1970,1975)$ in connection with his efforts to measure the circulation associated with aerodynamic surfaces in a wind tunnel. Schmidt's method will be discussed later but it is noted here that his apparatus was limited by the accuracy of time-of-flight measurements of the ultrasound waves travelling between an ultrasonic transducer and a microphone. During the past twenty years, significant advances in ultrasonic flow measurements have resulted in the development of electronic instrumentation capable of measuring the very small time differences associated with changes in the ultrasound wave propagation times. The technology has continually improved so that we are now able to measure volume flow rate and mean transverse velocities in pipes and conduits reliably and accurately in laboratory scale apparatus as well as in large industrial settings.

The propagation of sound through a moving fluid is increased in speed according to the component of fluid velocity along the propagation path, as integrated along the path. Thus, the average fluid velocity along the path can be measured in pipes and ducts, a fact utilized by the community interested in ultrasonic flow measurement. In this case, sound is transmitted along a path oblique to the flow and the difference in propagation time from the no-flow case is directly proportional 
to the volumetric flow rate. There are, of course, difficulties not the least of which is the variation of sound speed with temperature and thus spatially within the fluid. By propagating sound in both directions along the path, the effect of variation of sound speed can be nullified, a technique essential to precision flow measurement (Genthe and Yamomoto 1971). One of the inherent limitations of such measurements, has been the ability to measure very small time differences. Indeed, just determining the arrival of the ultrasonic pulse has been the subject of technological development as pioneered by the Westinghouse Leading Edge Flow Meter (LEFM) in the early 1970s. Relatively high ultrasonic frequencies are desirable because, then, the ambiguity of pulse arrival time is lessened. However, high frequencies are attenuated quickly in most fluids limiting the practical range. The timing problem has limited most flow measurement applications to short path lengths while the upper ultrasound frequency bound due to absorption results in continually lower frequencies being dictated for longer and longer path lengths.

In this paper, we present a brief overview of the developments in the field of ultrasonic flow measurements pertaining to vortex circulation, as well as a new scheme for accurate circulation measurement which circumvents limitations associated with the previous methods. The new method is verified by comparing the bound circulation of a NACA 0012 airfoil with that computed from the lift measurements. Other examples are also presented where the ultrasound method has been applied to the delta wing vortices and the free-surface vortex is a cylindrical tank.

\section{2}

\section{Technique}

To measure the circulation in a specified region of flow, the time integral of velocity along a closed path surrounding the region is required. Ultrasound methods can be used to measure such line integrals. For certain applications, a single straight ultrasonic path can be utilized. The principles for this class of measurements are described first. Then, the more general method of employing a closed path within the flow field is delineated.

\section{1}

\section{Straight path}

Since sound waves travel through fluids with a speed which is the sum of the local sound speed and the local flow velocity component in the direction of wave propagation, sound waves can be utilized to measure the line integral of velocity along a given path. Schmidt (1975) utilized this principle to determine the circulation, $\Gamma$, of a vortex by propagating sound along a single path through the induced velocity field of a potential vortex, as in Fig. 1. The propagation time between transducers 1 and 2 can be related to the sound speed $a$ and the local flow velocity component along the path $V$ as follows:

$T_{1 \rightarrow 2}=\int \frac{\mathrm{d} l}{a+V(l)}$

where $\mathrm{d} l$ is the line element along the path between the two transducers. If one assumes that the sound waves travel through the potential segment of the vortex, where the

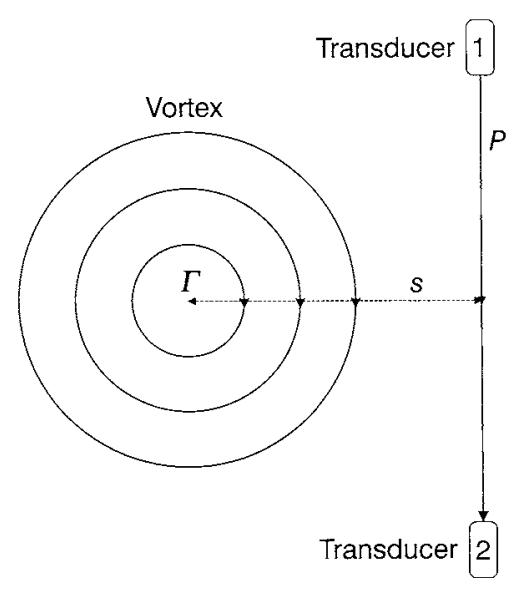

Fig. 1. Schematic of the straight ultrasonic path between two transducers. The distance between the two transducers is $P$ and that between the vortex center and the ultrasound path is $s$

tangential velocity is $\Gamma / 2 \pi r$, then $T_{1 \rightarrow 2}$ can be related to the vortex circulation.

$T_{1 \rightarrow 2}=\frac{P}{a}-\frac{\Gamma s}{\pi A a^{2}} \tan ^{-1}\left(\frac{P}{2 A}\right)$

Here $A=\left[s^{2}+(\Gamma s / 2 \pi a)\right]^{1 / 2} ; s$ is the distance between the vortex center and the propagation path and $P$ is the distance between the two transducers. Note that the propagation time is shorter than that for a fluid at rest. For sound waves traveling from transducer 2 to 1 , the transit time would be

$T_{2 \rightarrow 1}=\frac{P}{a}+\frac{\Gamma s}{\pi B a^{2}} \tan ^{-1}\left(\frac{P}{2 B}\right)$

with $B=\left[s^{2}-(\Gamma s / 2 \pi a)\right]^{1 / 2}$. Schmidt also observed that, if two pairs of transducers are utilized such that sound beams are propagating in opposite directions (counter propagation), then the difference between the two propagation times can be expressed as

$\Delta T=T_{2 \rightarrow 1}-T_{1 \rightarrow 2} \cong \frac{2 \Gamma}{\pi a^{2}} \tan ^{-1}\left(\frac{P}{2 s}\right)$.

The above expression assumes that the vortex circulation is much smaller than $2 \pi a s$. In the case of a planar airfoil in wind tunnel, the assumption amounts to the requirement of having a freestream Mach number much smaller than $4 \pi / C_{l}$. This condition is satisfied in all low speed tunnels. The effects of compressibility will be discussed later. Furthermore, if the setup is designed such that $P \gg 2 s$, then $\Delta T$ is simply the ratio of vortex circulation to the speed of sound squared. Thus, it can be seen that the circulation is directly proportional to the difference in propagation times between the two transducers.

By placing two pairs of transducers (transmitting in opposite directions) behind an airfoil in a wind tunnel, Schmidt (1975) measured the bound circulation as a function of angle of attack and freestream velocities up to $30 \mathrm{~m} / \mathrm{s}$. The circulation increased linearly with the angle of attack (up to a critical value) as well as the freestream velocity. These trends clearly verified the applicability of this ultrasonic technique for 
circulation measurements around airfoils. Weber et al. (1995) utilized a setup similar to Schmidt's to measure the circulation around a dynamically pitching airfoil. That study proved that transit time differences as small as 100 nanoseconds can be measured and the ultrasonic method is capable of characterizing circulation in time-varying flows.

Schmidt's developments presumed that the vorticity was all contained within a compact region such that the overall flow field behaved in a known manner; namely, the potential vortex. Moreover, the precise location of the vortex core is required if the distance between the two transducers is not significantly greater than that between the vortex core and the ultrasonic path. These difficulties amount to minor errors in well-behaved flows with a stationary vortex such as that associated with the bound circulation of airfoils. On the other hand, in cases where the velocity distribution is not known or the vortex is free to move in space, the just-described method suffers diminished accuracy. The situation is even less clear in cases where multiple vortices exist in the flow field. The measured time differences cannot be directly related to the circulation of each individual vortex without making further assumptions.

\section{2}

\section{Closed path}

The method described herein, while based on Schmidt's work, overcomes the above difficulties. The method utilizes ultrasonic pulses that travel in a continuous closed path around the vortex. The closed path can be achieved by the reflection of ultrasonic pulses from the interior surfaces of a duct or by reflectors placed within the flow, as shown in Fig. 2. The reflection follows Snell's law since narrow ultrasonic pulses behave substantially as geometric rays with divergence angles of only a few degrees. The time of travel between the transmitter and the receiver is measured accurately for pulses traveling in the direction of vortex rotation and against it. As it is shown below, the difference between the two travel times is linearly proportional to the calculation contained within the closed path. By varying the area enclosed by the closed path, the distribution of circulation can be found as well. Improvements in ultrasonic flowmeter technology have enabled measurement of time differences of the order of few nanoseconds such that circulation measurements can be made in laboratory scale experiments involving liquids.

The ultrasonic circulation measurement technique consists of measuring the transit time of ultrasonic pulses traveling in the sense of vortex rotation $\left(T_{\text {down }}\right)$ and opposite to it $\left(T_{\mathrm{up}}\right)$. Then,

$T_{\text {down }}=\oint \frac{\mathrm{d} l}{a+V(l)} \quad$ and $\quad T_{\text {up }}=\oint \frac{\mathrm{d} l}{a-V(l)}$

where $V(l)$ is the local fluid velocity component along the closed path. By taking the difference between these two transit times, an expression for the line integral of velocity along the closed path can be obtained as follows:

$\Delta T=T_{\text {up }}-T_{\text {down }}=2 \oint \frac{V(l) / a^{2}}{1-(V / a)^{2}} \mathrm{~d} l \cong \frac{2}{a^{2}} \oint V(l) \mathrm{d} l$

Therefore, circulation is linearly proportional to the transit time difference $\Delta T$ as long as the ratio $(V / a)$ remains small, say

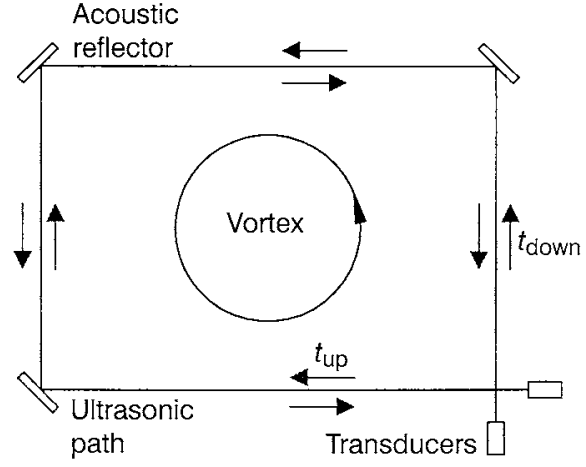

Fig. 2. Schematic of a possible closed path setup for circulation measurement

less than 0.1 . This condition is invariably satisfied for aqueous flows and low speed flows in air especially since $V$ is the velocity component along the closed ultrasonic path and not necessarily the freestream velocity. If the two transit times are added together, then the sum of line integrals results in the total ultrasonic path length $P$ divided by the local sound speed

$\Sigma T=T_{\text {up }}+T_{\text {down }}=\frac{2}{a} \oint \frac{\mathrm{d} l}{1-(V / a)^{2}} \cong \frac{2}{a} P$

This relation is quite helpful since the local sound speed may not be known at any given time instant. Once the total path length $P$ is known as well as the two transit times, both the local sound speed as well as the circulation can be calculated. Since circulation $\Gamma$ is the line integral of velocity around a closed path, the transit time difference relation simplifies to

$\Gamma \equiv \oint V(l) \mathrm{d} l=0.5 a^{2} \Delta T=0.5\left(\frac{2 P}{\Sigma T}\right)^{2} \Delta T=2\left[\frac{P}{\Sigma T}\right]^{2} \Delta T$

This last expression allows for the direct computation of circulation from accurately measurable transit time difference $\Delta T$ 's and $\Sigma T$ 's without the need for any calibration constant or the knowledge of the local sound speed. It is noteworthy that the closed path should lie in a plane perpendicular to the vortex axis. This method of circulation measurement has several advantages over the straight path described earlier. The precise location of the vortex relative to the ultrasonic path is not needed. The technique measures the total circulation enclosed by the path. Moreover, a priori knowledge of the vorticity distribution or the induced velocity behavior is not assumed in the closed path scheme. The issue of multiple vortices in the flow field is also resolved because the measured time difference is linearly proportional to the algebraic sum of all the vortex circulations enclosed by the path.

There are several issues regarding the circulation measurements that need to be addressed here. Although the requirement that the local fluid velocity $V$ be small in comparison with the local speed is satisfied in the practical aqueous flows, velocities in air flows may be comparable with the sound speed. In such cases, the denominator in the integral of Eq. (6) does not reduce to unity. In fact, if on average $V \approx a$, then the 
integral becomes singular. Thus, for this reason and those associated with the modification of sound waves in compressible flows, the closed path circulation measurement technique is unlikely to be applicable to compressible flows. At the same time, if $0.1<V / a<0.3$, the measured $\Delta T$ would still be linearly related to $\Gamma$ but with an additional constant to be determined after the mean velocity component along the path is found.

The variations of temperature and sound speed along the ultrasound path is another concern because we assumed that the sound speed was constant in the approximation of Eq. (6). Two factors help reduce the dependence of measured circulation on the sound speed variations. First, by propagating the sound in clockwise and counter clockwise directions and using Eq. (8) instead of (6) for calculating $\Gamma$, the effects of sound speed variations along the closed path would be minimized. Moreover, temperature variations as much as $\pm 3{ }^{\circ} \mathrm{C}$ would only alter the measured circulation $\left(\sim a^{2}\right)$ by $1 \%$ at room temperature air. Thus, as long as temperature variations do not significantly change the sound speed, the measured circulation should be free from temperature effects.

Sound pulses travel with a velocity that is the vector sum of the local flow velocity and the sound speed along the propagation direction. Therefore the true path of sound waves within a velocity field has to be found from ray-tracing methods (Boone and Vermaas 1991). The velocity components in the plane of the closed path result in curved paths. As an example, the path of sound waves due to a simple free vortex is shown in Fig. 3. The resultant path indicates that the change in the transit time of ultrasound pulses between the two transducers is a result of the elongation of the path as well as the flow-induced increase of the propagation speed. However, by subtracting the transit time of counter-propagating pulses the changes in $\Delta T$ due to the elongation of the path length would be removed. The path length elongation is independent of the travel direction of sound pulses. This is another reason for the use of counter-propagating pulses.

The flow velocity component perpendicular to the closed path shifts the sound pulses in the downstream direction. This may result in the transmitted pulses never reaching the receiver if the shift is larger than the size of the receiver sensor. The spatial extent of the downstream shift is given by the mean transit time multiplied by the velocity component normal to the path. If the sound pulses are transmitted in one direction between the two transducers at all times, the arrival of the transmitted pulses can be ensured by shifting the receiver with respect to the transmitter. On the other hand, relative shifting of the transducers would not be useful if counter-propagating pulses are employed. The problem is resolved by the natural broadening of narrow sound pulses as they travel through the fluid or using lenses to form a diverging beam. For example, common ultrasound transducers for use in air create pulses that expand conically at a half angle of about $3^{\circ}$. Thus the transmitted pulses in a flow field that are shifted by an amount less than the width of the broadened pulses would be 'heard' by the receiver. The broadening of the sound pulse would not affect the circulation measurements since the arrival time and not the amplitude of the pulse is required for the calculation of transit time differences. Most commercial ultrasonic flowmeters utilize this effect to make accurate measurement of the volume flow rate in pipes and conduits. Yeh and Mattingly

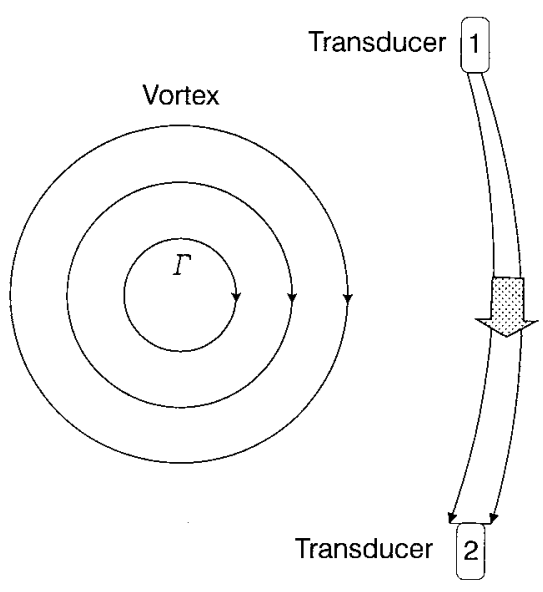

Fig. 3. The path traced by an ultrasound pulse traveling from transducer 1 or 2 . The curvature is caused by the induced velocity of the vortex. The broadening of the pulse is indicated by the increasing width of the curved path

(1997) performed a series of computations to observe the effects of various velocity fields on the path traced by ultrasound pulses. A ray-tracing method was utilized in these computations and they were primarily concerned with the accuracy of ultrasonic flowmeters. They concluded that the flow-induced curvature of the sound paths can be ignored, i.e. less than $0.5 \%$ change in the measured $\Delta T$, as long as the spatially-averaged velocity affecting the path is less than $10 \%$ of the sound speed. This restriction is similar to that imposed by the approximation in Eq. (6).

The above discussion directly impacts the spatial extent of the circulation measurements. If the area enclosed by the path is a critical parameter, then the effects of curved paths and pulse broadening would have to be considered in the calculations of the enclosed area. Alternatively, if the flow contains well-defined vortices and only the integrated circulation is of interest, then the variations in the path can be ignored. In any case, by requiring the flow velocities to be no larger than $10 \%$ of the sound speed, the variations in the path from the straight lines would be quite small.

One of the advantages of the ultrasound technique becomes evident in cases where time-dependent measurements of the circulation are required. The temporal resolution of an ultrasound system is limited by the time it takes the pulses to travel around the path. Thus, for a path length of approximately $1 \mathrm{~m}$, the sum of the transit times in the two directions would be on the order of $6 \mathrm{~ms}$ in air at room temperature. This translates to a sampling rate of about $100 \mathrm{~Hz}$, assuming a $2 \mathrm{~ms}$ time between 'hearing' a pulse and then firing of the subsequent pulse. Shortening the path length improves the maximum sampling rate. Since the transit times are much shorter in liquids, higher sampling rates would be possible. Furthermore, circulation measurements, which only involve the algebraic manipulation of transit times, can be acquired in real-time thus allowing the implementation of ultrasound sensors for active control systems.

Although ultrasonic imaging techniques have also been utilized in the past (Trebitz 1982) to measure the velocity 
field of vortices in laboratory scale flows, local temperature variations can strongly affect the outcome of these measurements. For this reason and the associated question of scaling in large (industrial) settings, it is believed that the abovedescribed time-of-flight technique may be preferable in certain applications where only the integrated measurements are desired. For example, acoustic transmission of very low frequency sound waves have been used to measure the mean fluxes and sound speeds in the oceans (Munk 1986). Such measurements must take into account the variation of sound speed with depth and path curvatures, and cannot exploit the simplifications afforded by the narrow high-frequency sound pulses utilized here.

\section{3}

\section{Hardware}

The choice of transducer frequency, path length, and the electronics for different applications is dictated by several parameters. Of primary concern is the arrival of detectable pulses at the receiving transducer. Higher ultrasound frequencies are desired because leading edge detectors would have reduced errors in recording transit times. On the other hand, the attenuation of sound waves by the fluid becomes increasingly significant at higher frequencies. Hence, the maximum path length is governed by the ultrasound frequency and the fluid attenuation properties.

In our laboratory, we have used ultrasonic transducers with a frequency of $100 \mathrm{kHz}$ for air applications. These transducers have a diameter of about $2 \mathrm{~cm}$ and have been successfully utilized for path lengths up to $2 \mathrm{~m}$. Path lengths as large as tens of meters have been used for mean flow measurements in industrial stacks. We have used both commercial flowmeters as well as in-house electronics coupled with a $10 \mathrm{MHz}$ counter for the transit time measurements. The commercial flowmeter (Parametrics Model GP68) has been used for steady flows and has better resolution and accuracy for $\Delta T$ measurements when compared to our in-house system. On the other hand, our in-house system is capable of making circulation measurements in unsteady flows. The uncertainty in $\Delta T$ measurements with the commercial flowmeter is of the order of ten nanoseconds, whereas our in-house system is limited to a minimum of $0.1 \mu \mathrm{s}$. Therefore, the in-house system would be more appropriate for relatively larger circulation values. Our experience has shown that a minimum $\Delta T$ of $50 \mathrm{~ns}$ can be measured with a path length of approximately $2 \mathrm{~m}$. This translates to an uncertainty of $\pm 0.003 \mathrm{~m}^{2} / \mathrm{s}$ in circulation for air at room temperature.

For aqueous flows, transit times are smaller by a factor of about 5 in comparison with air applications. Higher ultrasound frequencies are necessary to ensure equivalent $\Delta T$ uncertainties. At the same time, high ultrasound frequencies are attenuated more rapidly thus limiting the maximum path length. As a compromise we have used $1 \mathrm{MHz}$ transducers in aqueous applications. The $1 \mathrm{MHz}$ transducers create pulses that can be detected at a distance of the order of $1 \mathrm{~m}$. We have operated these transducers in conjunction with a commercial liquid flowmeter (Panametrics Model 6068) for path lengths up to $50 \mathrm{~cm}$.

In order to reflect the ultrasonic pulses, a material with acoustic impedance, i.e. density multiplied by the sound speed $\rho a$, much different from the fluid is needed. Typically, solids such as metals easily satisfy this requirement for both air and water applications. For external flows, we have used smooth surface stainless steel plates with dimensions comparable to the sound pulses $(\sim 2-3 \mathrm{~cm})$. For internal flows such as in cylindrical pipes, the internal walls can be utilized for reflecting the pulses as long as the pipe material has acoustic impedance appreciably greater than the fluid. Examples of the ultrasonic setups utilizing these reflectors and other hardware are described in the next two sections.

\section{3}

\section{Verification}

An experiment was setup to confirm the validity of the direct circulation measurement technique via the closed path and to assess the accuracy of the measured circulation values against independent means. The bound circulation of a rectangular NACA 0012 airfoil at its mid-span was measured in a wind tunnel at various angles of attack. The lift generated by the airfoil was also measured simultaneously by a pair of load cells at the two ends of the airfoil. Since the airfoil spanned the entire tunnel height, the flow was assumed two-dimensional except near the two ends. The airfoil lift $L$ can be related through the Kutta-Joukowski theorem to the bound circulation. By comparing the circulation measured directly via the ultrasound system and comparing that value to that computed from the lift measurements $\left(L / \rho_{\infty} U_{\infty} b\right)$, the accuracy of the system can be evaluated.

The airfoil had a chord length $c$ of $10.5 \mathrm{~cm}$ and a span $b$ of $45 \mathrm{~cm}$. The angle of attack $\alpha$ was measured by a rotary position sensor. The airfoil surface was smooth and the boundary layers were not tripped. The tunnel blockage ratio was kept below $5 \%$ to avoid having to employ empirical lift corrections. The tunnel was run at freestream velocities $U_{\infty}$ of $7.1,10.0$, and $15.0 \mathrm{~m} / \mathrm{s}$ resulting in airfoil Reynolds numbers $\left(U_{\infty} c / v\right)$ of $4.8,6.8$, and $10.2 \times 10^{4}$, respectively. The freestream turbulence level in the tunnel was $0.9 \%$. The angle of attack was varied from $-4^{\circ}$ to $10^{\circ}$ in $2^{\circ}$ increments. Further details regarding the experimental setup can be found in Desabrais (1997).

The ultrasound path chosen for this experiment was a triangle perpendicular to the axis of the airfoil, as shown in Fig. 4. The triangular path was chosen to keep the number of reflectors to a minimum and to keep the total path length relatively short. The path length $P$ in this setup was $201 \mathrm{~cm}$. A pair of $100 \mathrm{kHz}$ ultrasound transducers was placed in a cavity on one of the tunnel sidewalls. Two acoustic reflectors made of stainless steel plates were employed to redirect the sound pulse. The reflector opposite the transducers was also placed in the tunnel sidewall such that neither this reflector nor the transducers interfered with the flow in the tunnel. The second reflector was placed at a distance of 3 chord lengths behind the airfoil. Although this reflector was in the wake of the airfoil, it was sufficiently away from the airfoil not to disturb the flow there. Moreover, the flow separation around this reflector imparts negligible changes to the circulation within the closed path. It is noteworthy that only the vorticity in the airfoil boundary layers contribute to the mean measured circulation. At the trailing edge, the mean flux of vorticity from the upper and lower surfaces of the airfoil balance out and there is no mean contribution from the wake. Thus the 


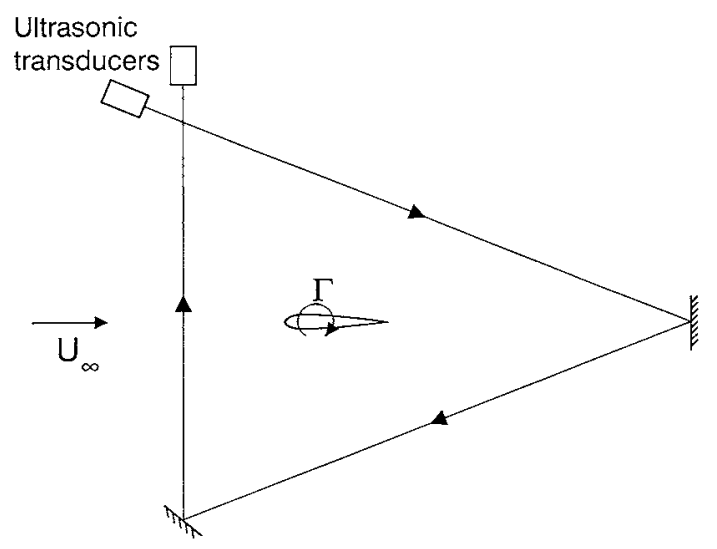

Fig. 4. The triangular ultrasound path used in the airfoil experiments

placement of the second reflector is immaterial for the circulation measurements.

Each transducer acted as a transmitter as well as a receiver. The difference in transit times along the path in the clockwise and counter clockwise directions were measured and over 100 samples were averaged for each angle of attack and freestream velocity. The circulation was calculated by multiplying $\Delta T$ by $a^{2} / 2$. Calibration of the flowmeter in still air resulted in minimum measurable transit time differences corresponding to $\pm 0.003 \mathrm{~m}^{2} / \mathrm{s}$ of circulation at room temperature. At an angle of attack of $2 \circ$ and a freestream velocity of $7.1 \mathrm{~m} / \mathrm{s}$, this error amounts to $2 \%$ of the measured circulation while at $\alpha=10$ and $U_{\infty}=15.0 \mathrm{~m} / \mathrm{s}$, the error is only $0.4 \%$ to the measured value. Thus, the random errors are less than $2 \%$ in the measured data.

Initially a series of measurements were made without the airfoil in the tunnel to remove the effects of any residual circulation in the tunnel, including that due to the boundary layers on the sidewalls and that generated by the second reflector. The mean values of the residual circulation were $0.012,0.007$, and $-0.024 \mathrm{~m}^{2} / \mathrm{s}$ at the $7.1,10.0$, and $15.0 \mathrm{~m} / \mathrm{s}$ freestream velocities, respectively. These amounts were subsequently subtracted from the bound circulation measurements at the corresponding freestream velocities.

The measured lift coefficient $C_{L}\left(\equiv 2 L / \rho_{\infty} U_{\infty}^{2} c b\right)$ from the load cell data as well as the equivalent values from circulation measurements $\left(2 \Gamma / U_{\infty} c\right)$ are plotted against the airfoil angle of attack in Fig. 5. The vertical error bars indicate \pm one standard deviation of the measured data; the random errors are significantly smaller than these values. All the data correspond to the pre-stall regime. Due to the relatively low Reynolds number of the flow, the lift coefficient data do not quite follow a straight line. In general there appears to be good agreement between the lift coefficients resulting from the ultrasound data and the load measurements.

To assess the accuracy of the direct circulation measurement technique, the measured mid-span circulation is plotted against that obtained from the lift measurements, i.e. $L / \rho_{\infty} U_{\infty} b$, in Fig. 6. All the data from Fig. 5 plots are included in Fig. 6. A regression line was fitted to the data; the correlation coefficient was better than 0.99 . The slope of the regression line, which should ideally be unity, is 1.06 . This indicates that the overall linearity of the ultrasound measurement device with this setup was $6 \%$. It is expected that the technique is even more accurate because the circulation from the lift measurements is as average across the entire span of the tunnel, containing the end regions where horseshoe vortices alter the flow field. In fact, the regression line fitted to the data from each individual freestream velocity yields slopes of only $3 \%$ to $4 \%$ greater than unity. Consequently, it was concluded that the closed path technique of circulation measurement results in measurements that the accurate to within $5 \%$. Two applications employing the closed path ultrasonic technique in air and water flows are described next.

\section{4}

\section{Applications}

There are several applications that can take advantage of an ultrasonic instrument for circulation measurements. For example, the swirl strength in pipes and closed conduits can readily be measured with clamp-on ultrasonic transducers arranged in a manner comparable to the prescribed closed path method. The prediction of bound circulation associated with different sections of modern high lift, swept wings and the circulation associated with aircraft wing tip vortices has been of paramount importance since the advent of large commercial airliners. Information on circulation distribution on a wing can assist with the optimization of performance since force measurements using load cells only indicate the combined loads on the wing and not the distribution of the load.

Moreover, airport traffic control and flight safety issues can be handled in a quantitative manner if the local vortex strengths in the wake of aircraft are known. We have applied the closed path ultrasound technique to two flow fields in which the circulation associated with the air flow over a delta wing and the free-surface (bath-tub) vortex in a cylindrical container were measured. These efforts are described below.

\section{1}

\section{Delta wing vortex}

The flow about delta wings is characterized by a pair of large vortices resulting from the separation of the flow over the leading edges. Beyond certain angles of attack, these concentrated vortices breakdown or 'burst' into larger turbulent vortices. Although several studies have measured the velocity field of these vortices using hot-wires and LDV, they have been most effective in the pre-burst regime. The vortices in the post-burst regime are quite sensitive to the presence of physical probes and the possible meandering of the vortices tends to reduce the accuracy of LDV measurements. We have utilized the ultrasound technique to map the circulation of these vortices as a function of the chordwise location, angle of attack, and for two wing sweep angles of $60^{\circ}$ and $70^{\circ}$. Both the pre-burst and post-burst regimes were examined.

The ultrasound path in this setup, shown in Fig. 7, was a rectangle of $30.5 \times 20.5 \mathrm{~cm}$ that was always perpendicular to the root chord line. Half-delta wings mounted on a false wall were used in these experiments. A pair of $100 \mathrm{kHz}$ transducers along with the flowmeter used in the airfoil study was employed to generate the pulses and to measure the transit times. Three reflector were used to direct the ultrasound 

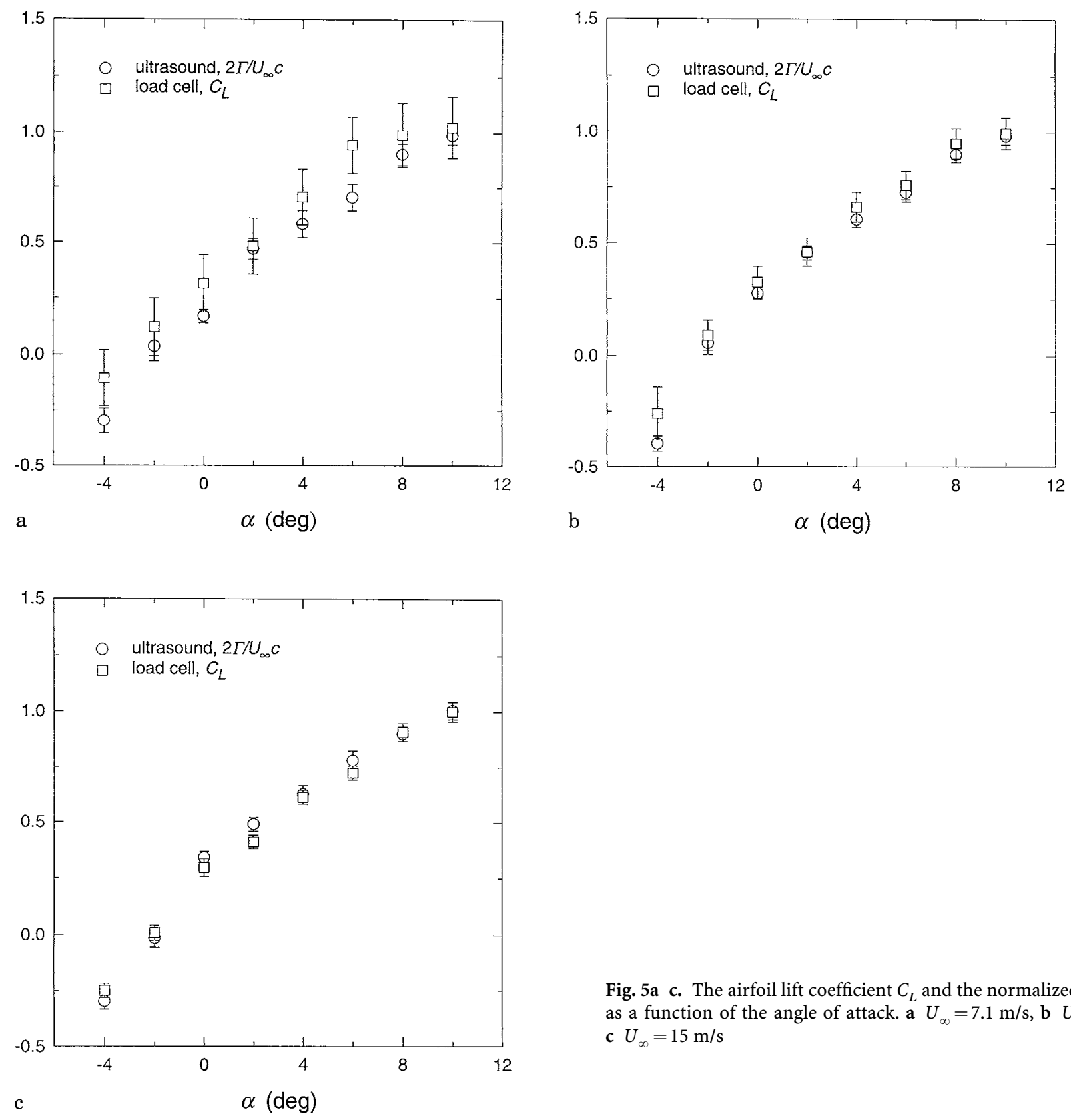

Fig. 5a-c. The airfoil lift coefficient $C_{L}$ and the normalized circulation as a function of the angle of attack. a $U_{\infty}=7.1 \mathrm{~m} / \mathrm{s}, \mathbf{b} U_{\infty}=10 \mathrm{~m} / \mathrm{s}$, c $U_{\infty}=15 \mathrm{~m} / \mathrm{s}$

pulses. These reflectors were placed sufficiently away from the delta wing so as not disturb the flow around the wing. One of the transducers, which was mounted into the false wall supporting the half-wing, emitted sound pulses along the wing surface while the other transducer emitted pulses normal to the surface and along the false wall. The centers of the transducers were approximately $1 \mathrm{~cm}$ away from the wing and false wall surfaces. This arrangement precluded the circulation associated with the boundary layers on the false wall and the secondary separation on the half-wing from being accounted in our measurements. The uncertainty in the $\Delta T$ measurements translated to an uncertainty in circulation of $\pm 0.003 \mathrm{~m}^{2} / \mathrm{s}$. This is $5 \%$ of the smallest and $0.2 \%$ of the largest measured circulation values associated with the delta wings in our setup. It is noteworthy that our measurements of circulation

encompassed the entire flow region between the wing surface and the freestream, thus all the vorticity in the enclosed region would contribute to the measurement irrespective of the vortex core location.

The experiments were run in a wind tunnel at a freestream velocity of $11.2 \mathrm{~m} / \mathrm{s}$. Both the $60^{\circ}$ and $70^{\circ}$ half-wings had root chord lengths of about $25 \mathrm{~cm}$ resulting in a chord Reynolds number of $1.9 \times 10^{5}$. Both wings had a thickness of $2.3 \mathrm{~mm}$ and the leading edges were beveled toward the lee side. Circulation of the vortex at $35 \%, 50 \%, 65 \%$, and $80 \%$ of the chord length was measured. The angle of attack $\alpha$ ranged from $2^{\circ}$ to $38^{\circ}$ for the $60^{\circ}$ wing from $2^{\circ}$ to $52^{\circ}$ for the $70^{\circ}$ wing. The maximum angles of attack were chosen so that the vortex burst occurred near the wing tip at the highest $\alpha$. The blockage ratio was kept below $3 \%$ for all cases. 


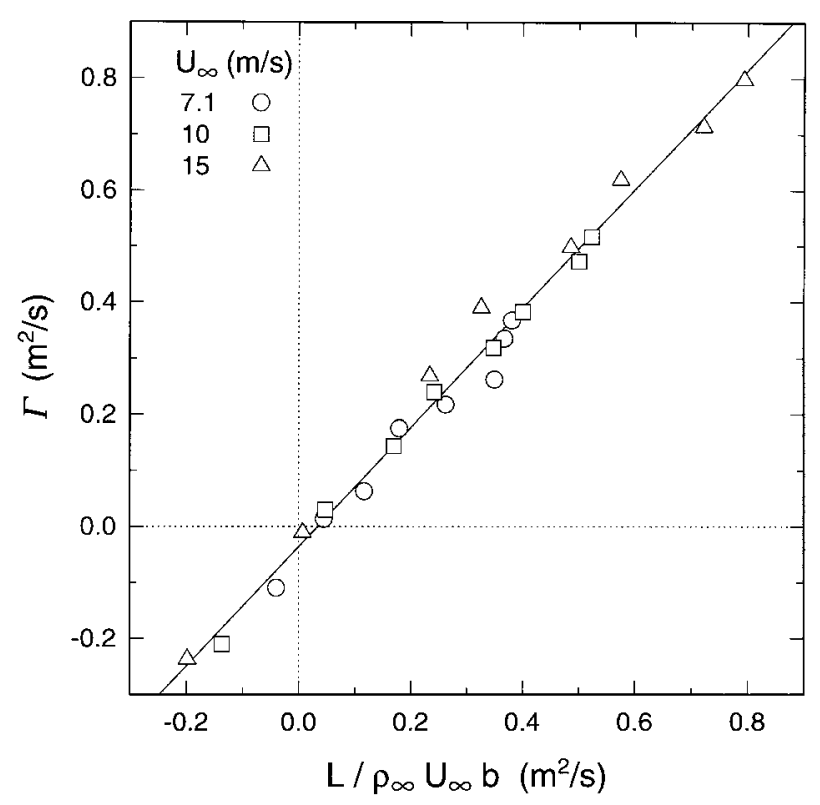

Fig. 6. Dependence of measured circulation on the circulation derived from the lift data

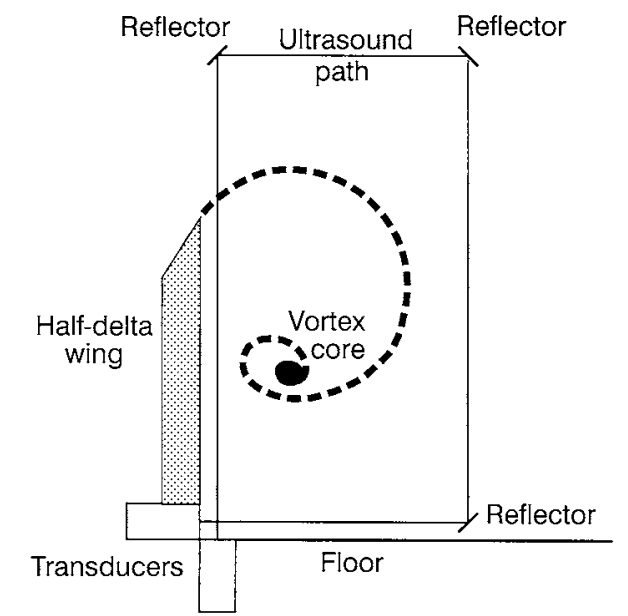

Fig. 7. Schematic of the ultrasound path used in the delta wing experiments

The circulation of the vortex at the $65 \%$ chord location of the $70^{\circ}$-wing was measured at several freestream velocities up to $15 \mathrm{~m} / \mathrm{s}$ at $26^{\circ}$ angle of attack. These measurements exhibited the expected linear dependence of the circulation of the freestream velocity. The circulation, normalized by the freestream velocity and the root chord length, is plotted against the angle of attack and the measurement station along the wing in Fig. 8. The data, which were average of 100 samples, agreed well with the previous (limited) measurements of circulation in the pre-burst regime. The present measurements further revealed that at any angle of attack the vortex circulation increases smoothly along the wing even through the burst region. At all measurement stations, the vortex circulation increased with the angle of attack up to a maximum value. At
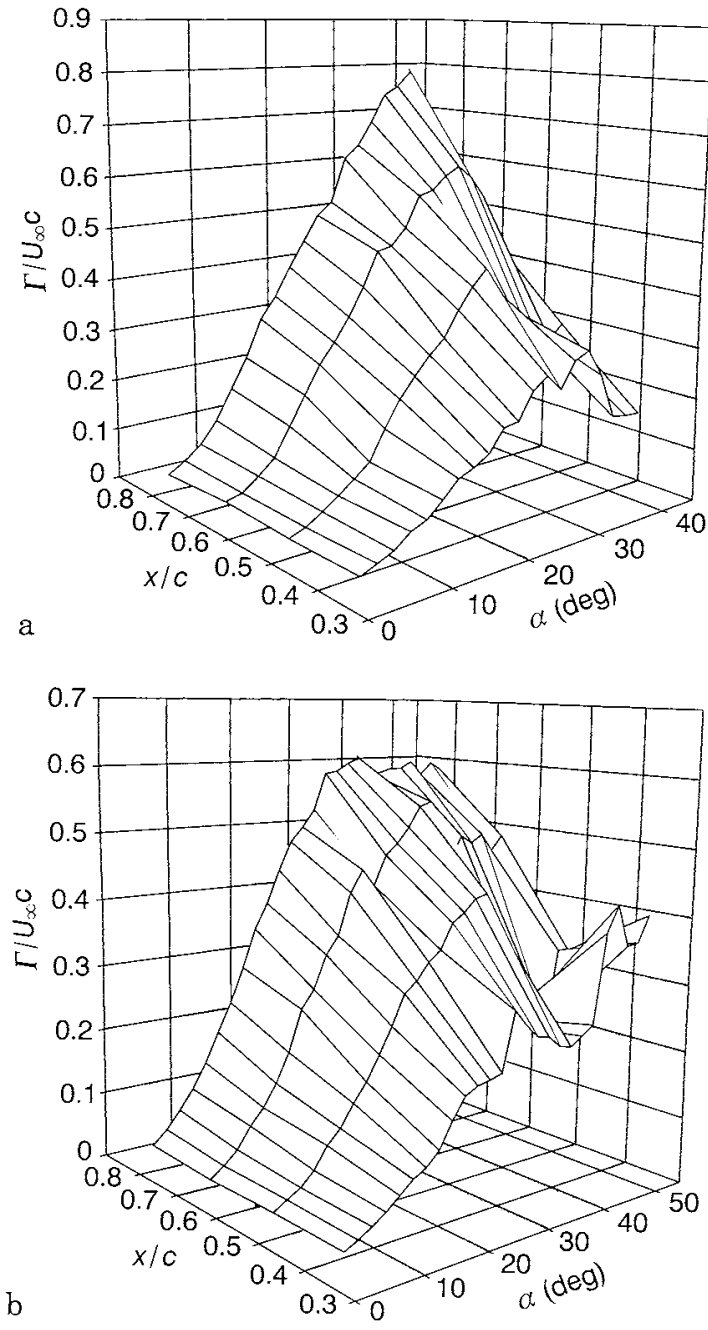

Fig. 8a,b. Circulation of the delta wing vortex as a function of the angle of attack and location along the root chord. a $60^{\circ}$ wing, b $70^{\circ}$ wing

larger angles of attack the circulation either remained constant or decreased slightly. Furthermore, the measured rms fluctuations of the circulation did not vary appreciably across the burst location indicating that the burst does not alter the mean value or the range of circulation magnitudes.

\section{2}

\section{Swirling free-surface flow}

The other application utilizing the closed path technique involved a free-surface (bath-tub) vortex in a cylindrical container. This problem is of particular interest in industrial settings because of the problems associated with air entrained into flow machinery. If the vortex strength can be correlated with the ability of the vortex core to entrain air, then an on-line monitoring system can be arranged to measure the vortex circulation directly and provide an alert when the flow conditions could result in air entrainment. Our experimental setup consisted of a water-filled cylindrical container which was open to the atmosphere and drained through a sharpedged centrally-located orifice on the bottom of the container. To maintain the water at the same height, water was pumped 


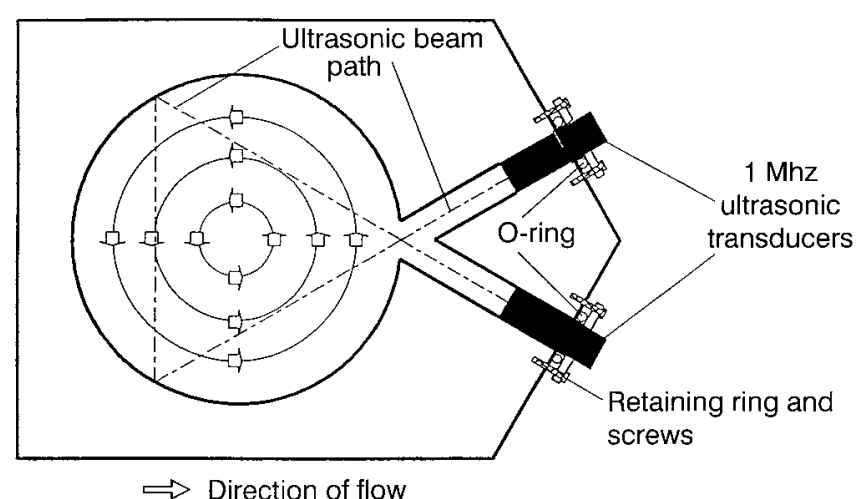

Fig. 9. Schematic of the ultrasound setup in the cylindrical container

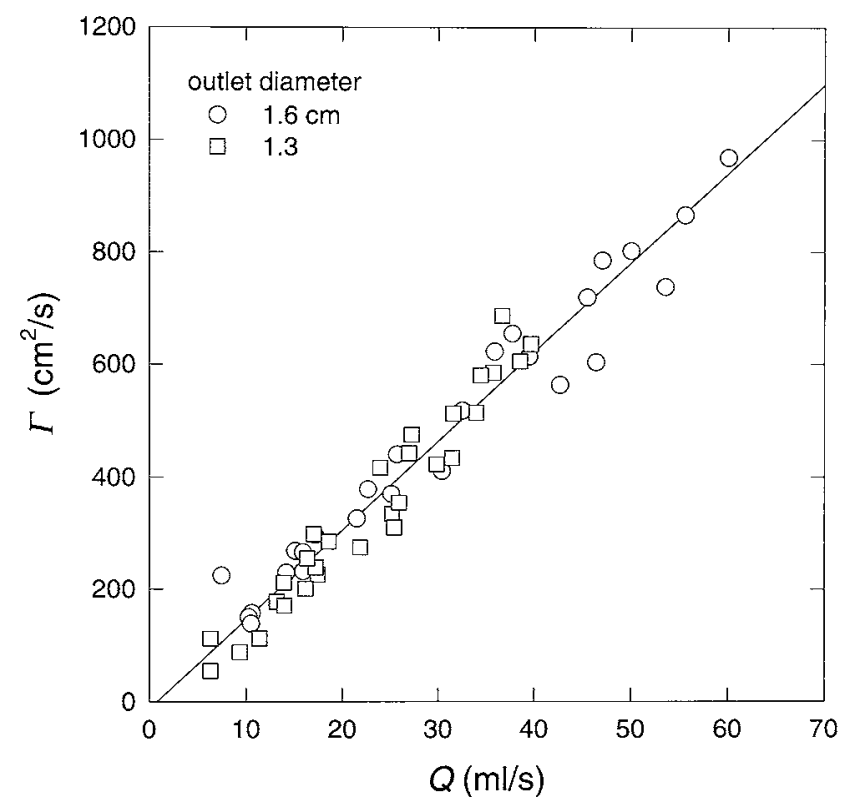

Fig. 10. Circulation of the free-surface vortex against volume flow rate

tangentially into the container through a small inlet beneath the free-surface. This arrangement created stationary freesurface vortices. The internal diameter of the container was $15.2 \mathrm{~cm}$ and the outlet orifice diameter varied from 0.32 to $1.6 \mathrm{~cm}$. The mean flow rate $Q$ through the system was monitored by a precision flowmeter.

The ultrasound path in the setup was a horizontal equilateral triangle tangent to the mid-radius circle. The path was located approximately halfway between the free-surface and the bottom. The path enclosed the central portion of the vortex, including any air column drawn through the core. The container walls were employed to reflect the ultrasound pulses. A pair of wetted $1 \mathrm{MHz}$ transducers were mounted such that the emitted pulses formed a $60^{\circ}$ angle with respect to each other. No extra reflectors were needed to re-direct the pulses. A schematic of the ultrasound path is shown in Fig. 9.

A commercial flowmeter (Panametrics Model PT868, on loan from Panametrics, Inc.) was used with this setup. The total sound path was $40 \mathrm{~cm}$ and the largest measured time difference
$\Delta T$ in our setup was $95 \mathrm{~ns}$. The uncertainty of circulation measurements was $20 \mathrm{~cm}^{2} / \mathrm{s}$, which corresponded to about $2 \%$ of the largest measured circulation in the present experiments.

The vortex circulation was measured as a function of the mean volume flow rate through the container for five different outlet diameters. Even though the vortex circulation is governed by the dimensionless parameters of the flow, e.g. Froude, Reynolds, and Weber numbers, they could not be varied independently. Changes in the volume flow rate would be reflected in the Reynolds number as well as the Froude number. Moreover, the free-surface level was not altered significantly to avoid changes in the container aspect ratio. Thus, the measured vortex circulation is presented as a function of the volume flow rate in Fig. 10 for the two outlet diameters of 1.3 and $1.6 \mathrm{~cm}$. Each data point is an average of 50 samples. It is anticipated that the vortex circulation would increase linearly with the flaw rate. The linear behavior is clearly discernible in these data and the correlation coefficient of the regression line is 0.98 . The slope of the best-fit line is $15.9 \mathrm{~cm}^{-1}$. A complete presentation of the data as well as a discussion of flow properties and scaling can be found in Smith et al. (1995).

The above examples were intended to show the versatility of the ultrasonic techniques for circulation measurements in a variety of settings. Besides these efforts, we have also measured the circulation distribution of wing tip vortices in a wind tunnel using the closed path technique. The results of that investigation will be presented elsewhere.

\section{5}

\section{Conclusions}

Ultrasonic methods can be utilized to set up inexpensive devices capable of directly measuring the circulation of vortical flows. The closed path technique provides the ability to measure the circulation in an enclosed area without prior knowledge of the vorticity distribution or the sound speed along the path. There are no calibrations involved with the ultrasonic methods, and the transducers and reflectors can be arranged such that they would not interfere with the flow field under investigation. The primary limitation of the closed path technique requires the velocity component along the closed path to be significantly smaller than the sound speed. This restriction limits the maximum velocities along the path to incompressible speeds in gaseous flows, whereas it is not a major concern in aqueous flows. The closed path technique was verified by comparing the measured bound circulation of a planar airfoil with that calculated from the lift measurements. The utility of the closed path technique was demonstrated by the measurements of circulation around delta wing vortices in air and free-surface vortices in water. The closed path technique opens up the possibility of employing ultrasonic measurement technology for on-line monitoring of circulation as well as introducing a global sensor for integration into advanced control systems.

\section{References}

Adrian RJ (1986) Multi-point optical measurements of simultaneous vectors in unsteady flow - a review. Int J Heat Fluid Flow 7: 127-145 Adrian RJ (1991) Particle-imaging techniques for experimental fluid mechanics. Annu Rev Fluid Mech 23: 261-304 
Boone MM; Vermaas EA (1991) A new ray-tracing algorithm for arbitrary inhomogeneous and moving media, inlcuding caustics. J Acoustic Soc Am 90: 2109-2117

Desabrais KJ (1997) Direct measurement of wing tip vortex circulation using ultrasound. M.S. thesis, Worcester Polytechnic Institute, Worcester, MA

Genthe WK; Yamamoto M (1971) A new ultrasonic flowmeter for flows in large conduits and open channels. In: FLOW: Its Measurement and Control in Science and Industry, Part 2, Flow Measuring Devices, (ed Wendt, RE Jr). Vol. 1, pp 947-955

Moreira J; Johari H (1997) Direct measurement of delta wing vortex circulation using ultrasound. AIAA paper 97-0743

Munk W (1986) Acoustic monitoring of ocean gyres. J Fluid Mech 173: 43-53

Schmidt DW; Tilmann PM (1970) Experimental study of sound-wave phase fluctuations caused by turbulent wakes. J Acoustic Soc Am 47: 1310-1324

Schmidt DW (1975) Acoustical methods for fast detection and measurement of vortices in wind tunnels. ICIASF '75 Record, pp 216-228

Smith RH; Durgin WW; Johari H (1995) The direct measurement of circulation in free surface vortices. AIAA paper 95-0104

Trebitz BO (1982) Acoustic transmission imaging for flow diagnostics. $\mathrm{PhD}$ thesis, California Institute of Technology, Pasadena, CA

Weber FJ; Durgin WW; Johari H (1995) Direct circulation measurements about a rapidly pitching airfoil using an ultrasonic system. AIAA paper 95-2269

Willert CE; Gharib M (1991) Digital particle image velocimetry. Exp Fluids 10: 181-193

Yeh TT; Mattingly GE (1997) Computer simulations of ultrasonic flow meter performance in ideal and non-ideal pipe flows. In: Proc 1997 ASME Fluids Engineering Division Summer Meeting, paper number FEDSM97-3012 